\title{
Efecto de la música sobre la respuesta cardiovascular en ejercicio de escolares entre 9 y 17 años*
}

\author{
Gustavo Gómez Becerra* \\ Jhon Freddy Ramírez Villada** \\ Andrés Felipe Ortiz Rico****
}

Recibido: diciembre 2 de 2016 • Evaluado: diciembre 15 de 2016

Aceptado: enero 5 de 2017

\section{Resumen}

Objetivo: analizar el efecto de la música sobre la respuesta cardiovascular en ejercicio de escolares entre 9 y 17 años.

Métodos: 74 individuos entre 9 y 17 años fueron agrupados en tres grupos (G1=9-11; G2=12-14; G3=15-17); participaron en prueba de esfuerzo con uso de la música (tratamiento 1) y sin ella (tratamiento 2). Para controlar el efecto de la música, fueron aplicados protocolos morfológicos (peso corporal, porcentaje graso, talla, índice de masa corporal) y fisiológicos (presión arterial y frecuencia cardiaca). Se estimó el esfuerzo de cada sujeto al final de la prueba (VO2).

" El proyecto fue presentado, avalado y respaldado por la Universidad Manuela Beltrán (Maestría en Ciencias y Tecnologías de la Actividad Física y el Deporte) durante el periodo 2015-2017. No hace parte de ningún grupo de investigación, fue un proyecto aprobado como propuesta libre.

** Maestría en Ciencias y Tecnologías de Deporte y la Actividad Física. Docente Universidad Manuela Beltrán (Bogotá, Colombia). Correo electrónico: licenciadoggomez@gmail.com

*** Pos-Doc. Ph. D. Docente Universidad de Antioquia (Medellín, Colombia). Grupo de Investigación en Actividad Física y Salud (AFIS). Correo electrónico: jhonramirezvillada@ gmail.com - jhon.ramirez3@udea.edu.co

"**** Profesor y magíster de la Facultad de Estadística, Universidad Santo Tomás (Bogotá, Colombia).Correo electrónico: andresortiz@usantotomas.edu.co 
Resultados: existen diferencias significativas ( $\mathrm{p} \leq 0.05$ y 0.01 ) entre los tratamientos (T1 vs. T2) en los grupos valorados (G1 vs. G2 vs. G3), y una tendencia reguladora, mas no significativa, en los indicadores de frecuencia cardiaca y presión arterial a favor del grupo T1.

Conclusión: la música tiene un efecto positivo sobre el control del estrés y permite mejorar el rendimiento físico. Los resultados tienen implicaciones positivas para la evaluación diagnóstica y el diseño de programas de actividad física para escolares entre 9 y 17 años.

Palabras clave: música, frecuencia cardiaca, presión arterial, consumo de oxígeno. 


\section{Effect of the Music on the Cardiovascular Response To Exercise of Students Between 9 - 17 Years Old}

Abstract

Objective: to analyze the effect of the music on the cardiovascular response to exercise of students between 9-17 old age. Methods: 74 individuals (917 years old) were grouped in three year intervals (G1=9-11; G2=12-14; G3=15-17) those who took part in an effort test with (treatment 1) and without (treatment 2) use of the music. To control the effect of the music, we measured morphological (corporal weight, oily percentage, height, index of corporal mass) and physiological variables (arterial pressure and cardiac frequency) and also, were estimated the effort of every subject at the end of the test (VO2). Results: There were significant differences ( $\mathrm{p}=0.05$ and 0.01 ) between the treatments (T1 vs. T2) per every group valued (G1 vs G2 vs. G3), and a slightly regulation, more not significant trend, in the indicators of cardiac frequency and arterial pressure for T1. Conclusion: The music has a positive effect for managing stress and allows to improve the physical performance. Those results have positive implications for the diagnostic evaluation and the design of programs of physical activity in students between 9-17 years old

Keywords: music, heart rate, blood pressure, xygen consumption. 


\section{Introducción}

La música influye en el sistema cardiovascular, haciéndolo más eficiente en el ejercicio. Las respuestas fisiológicas que genera son el resultado de cambios en el sistema nervioso autónomo desencadenados por el estímulo musical. El nervio simpático se activa por estímulos sonoros con un tempo rápido (Watanabe, Ooishi y Kashino, 2015); como resultado, la frecuencia cardiaca varía de acuerdo con el ritmo musical que se establezca durante el ejercicio (Karageorghis et al., 2011).

Música y ejercicio comparten procesos cerebrales. Por un lado, la percepción musical está basada en la organización temporal y tonal, donde participan cerebelo, córtex premotor y área motora suplementaria (Chen, citado por Soria-Urios, Duque y García-Moreno, 2011, p. 47). De igual forma, las respuestas al estrés psicofísico asociado al ejercicio intenso están mediadas por el hipotálamo. A su vez, estos intervienen en la actividad del sistema nervioso autónomo y en la respuesta fisiológica del sistema cardiovascular.

Un metaanálisis sobre los efectos de la música en diferentes signos vitales ha encontrado diferencias significativas en la presión arterial sistólica (PAS), la presión arterial diastólica (PAD) y la frecuencia cardiaca (FC), en los grupos que reciben estímulo musical, concluyendo que la reducción de la presión arterial y la frecuencia cardiaca inciden en los estados de ansiedad (Arazi, Asadi y Purabed, 2015). Al respecto Loomba, Arora, Shah, Chandrasekar y Molnar (2012) concluyen que hay una necesidad de ensayos controlados aleatorios más grandes que exploren la música como una intervención para reducir los parámetros fisiológicos como la PA y la FC, así como la ansiedad.

Se destaca la importancia del tempo de la música en la sincronización auditiva motora (Bood, Nijssen, Kamp y Roerdink, 2013). Pulsos que marcan tempos lentos, entre 95 y 100 pulsaciones por minuto (bpm, por el inglés beats per minute) y muy rápido (155-160 bpm), se equiparan a las intensidades del ejercicio programadas con el ritmo cardiaco (Karageorghis et al., 2011) Se crea, entonces, una interacción entre áreas motoras y áreas auditivas (Soria-Urios et al., 2011).

Por otro lado, la música produce un efecto relajante, retardando la respuesta anticipatoria cardiovascular. Zamorano, Peinado, Benito y Calderón 
(2013) indican que esta se manifiesta antes del inicio del ejercicio con un aumento del tono nervioso simpático, responsable de aumentar la FC y la PA. Así lo demostró el estudio de Jarraya et al. (2012), el cual verificó el efecto benéfico de la música durante el calentamiento en actuaciones supramáximos a corto plazo, al igual que un estudio realizado por Chtourou, Chaouachi, Hammouda, Chamari y Souissi (2012).

En concreto los estudios reportan los efectos reguladores de la música tanto en las respuestas fisiológicas cardiovasculares como en el rendimiento físico de grupos de deportistas de élite, amateur y recreacional. Sin embargo, faltan evidencias que establezcan con mayor precisión los efectos de la música en la respuesta cardiovascular anticipatoria, bajo esfuerzo físico y posesfuerzo en población diferente a la expuesta.

El propósito del estudio fue analizar los efectos que tiene la música en la respuesta cardiovascular en tres etapas: antes, durante y después del esfuerzo físico. Se implementó una prueba de esfuerzo submáximo aplicada a un grupo control y a un grupo experimental. Se incorporó música reproducida a una velocidad de 100-120 bpm a la prueba del grupo experimental y sin música a la prueba del grupo control y se analizaron la frecuencia cardiaca y la presión arterial como indicadores funcionales del sistema cardiovascular en el ejercicio. Como hipótesis, se establece que con el ejercicio programado con un tempo musical determinado, los valores de las respuestas cardiovasculares se regulan, mejorando el esfuerzo.

\section{Materiales y métodos}

El presente es un estudio cuasiexperimental orientado a evaluar la respuesta cardiovascular antes, durante y después del esfuerzo físico en dos grupos con la manipulación de la música acorde a las pulsaciones por minuto (G1: sin música, G2: con música) de escolares entre 9 y 17 agrupados por intervalos de edad (intervalo 1: individuos entre 9 y 11 años; intervalo 2: individuos entre 12 y 14 años; intervalo 3: individuos entre 15 y 17 años).

En la conformación de los grupos por intervalo de edad fueron considerados como criterios de inclusión individuos en edad escolar, de género masculino y femenino, entre 9 y 17 años, estrato social 4 y físicamente activos, de acuerdo con el formulario de nivel de actividad física PAQ -C / 
PAQA. Aun si reunían los criterios anteriores, fueron excluidos los sujetos que presentaban antecedentes familiares en primer grado como dislipidemias, hipertensión, diabetes, hipotiroidismo y cáncer, individuos con patologías cardiopulmonares, osteomusculares y hormonales, sujetos con un índice de masa corporal (IMC) superior a 25 y sujetos con contraindicaciones médicas para la realización de ejercicio. El estudio contó con la autorización del comité ético de las instituciones figurantes con registro N 08-200082014 y atendió los criterios sugeridos en la declaración de Helsinki vigente y actualizada desde el año 1964 hasta la fecha. En consecuencia, se les informó a todos los individuos de las características del estudio, sus objetivos, los procedimientos a aplicar, los riesgos potenciales y el uso de la información. Su participación fue aprobada mediante la firma de un representante mayor de 22 años, acorde a la ley nacional.

La selección de la muestra se realizó por conveniencia y es no probabilística, atendiendo a la naturaleza de los estudios cuasiexperimentales (Hernández, Fernández y Baptista, 2006). No obstante, fue considerada la distribución y control del error siguiendo una estrategia de distribución al azar de cada uno de los sujetos por grupo, es decir, cualquiera de los individuos tenía la misma posibilidad de pertenecer al grupo GE o GC por intervalo de edad.

Descripción de los procedimientos. Para dar mayor claridad y orden al apartado, las variables fueron agrupadas en variables de perfil general (peso, talla, porcentaje graso) y variables de tipo fisiológico (frecuencia cardiaca $[\mathrm{FC}]$ y presión arterial [PA]).

Para analizar la composición general se aplicó el método de bioimpedancia eléctrica (Alvero, Correas, Ronconi, Fernandez y Porta, 2010). Para la medición de las variables básicas de composición corporal se empleó un dispositivo marca Tanita ${ }^{\circledR}$, modelo BC-585F. El procedimiento del análisis de la composición corporal mediante el método de bioimpedancia eléctrica se realizó de la siguiente manera: la prueba se aplicó únicamente los miércoles entre 8:00 y 11:00 a.m. Los sujetos se presentaron en ropa deportiva, se tomó nota y registro de cada uno en el formato de recolección de los datos diseñado para este fin. Primero, se tomó la talla de acuerdo con el protocolo establecido (Sillero, 2005, p. 19). El tallímetro utilizado fue Health o Meter ${ }^{\circledR}$ Professional. Posteriormente, se ingresaron los datos solicitados por el sistema del monitor de composición corporal y se procedió a la toma de 
acuerdo con el protocolo seleccionado (Alvero et al., 2010). Finalmente, se registró en el formato de cada individuo el peso y el porcentaje graso.

En cuanto a las variables de tipo fisiológico, se tuvo en cuenta el test de Course-Navette, también denominado "test de ir y volver", diseñado por Léger y Lambert en el año 1982, y posteriormente modificado por Mercier, Godoury y Lambert (1988). Siguiendo las recomendaciones del protocolo, se acondicionó un coliseo deportivo cubierto. El audio de la prueba de Léger se reprodujo en un computador portátil marca Samsung® RV415, una consola y un amplificador. Se monitoreó la presión arterial (PA) y la frecuencia cardiaca (FC) de cada uno de los sujetos antes, durante y después de la prueba.

Para el registro de la FC antes, durante y después del esfuerzo físico con y sin música, fueron seguidos los procedimientos conforme al protocolo de medición y la utilización del pulsómetro marca Polar®, modelo FT1, de la siguiente manera: se colocó el dispositivo de acuerdo con el protocolo establecido y el manual de operación. Posteriormente, se le pidió a cada sujeto que ingresara a la pista de la prueba y se dieron las indicaciones referentes al test de Léger. El evaluador hizo la primera lectura de la FC y la registró en el formato, inició la prueba y en cada vuelta revisó la lectura del dispositivo. Tan pronto el sujeto alcanzó el 75 \% de la FCR, se hizo el registro y la prueba continuó. Cuando el sujeto finalizó la prueba, inmediatamente se registró su FC final y se activó el cronómetro para tomar el tiempo de recuperación. Se hizo registro a los 3 y a los 6 minutos para concluir el protocolo.

Por otra parte, la medición de la PA fue ejecutada con el tensiómetro digital pediátrico marca Riester®, siguiendo los procedimientos descritos por varios autores (Villegas González, Villegas Arenas y Villegas Gómez, 2012). La toma se hizo en cuatro momentos, el primero, 3 minutos antes de la realización de la prueba de Léger, la segunda al culminar la prueba, la tercera a los tres minutos y la cuarta a los seis minutos.

Respecto a la programación de la música (variable independiente), en la creación de las pistas musicales se empleó la clasificación AudioFuel, un conjunto de piezas musicales diseñadas para ayudar a correr (Lane, Davis y Devonport, 2011), con la aplicación Run On Beat, diseñada para correr con música ajustada al ritmo de carrera. Para el estudio se elaboró una lista de 10 canciones pop y rock grabadas a una velocidad de 100 a 120 bpm (música cuyo tempo fue clasificado como moderato - allegro (Núñez Fernández, 
2009). La lista de canciones se descargó y se reprodujo en un computador portátil marca Samsung® RV415, una consola y un amplificador.

En la valoración de las muestras se reprodujo el listado de canciones de manera aleatoria 5 minutos antes de la prueba de Léger e inmediatamente finalizada la misma, de manera que la señal de audio para el cambio de ritmo fuera escuchada. Al finalizar el protocolo y durante la toma de la frecuencia cardiaca y presión arterial, nuevamente se continuó con la música.

\section{Resultados}

El comportamiento de la frecuencia cardiaca $(1 / \mathrm{min})$ antes, durante y después de la prueba de esfuerzo (prueba de Course-Navette), de los grupos G1 y G2, es presentado en la figura 1. Se resalta que los valores promedio de la frecuencia cardiaca antes de la prueba y la frecuencia cardiaca de recuperación (3 minutos después de finalizada la prueba) variaron entre G1 y G2, aunque no de manera significativa.

Figura 1. Valores de frecuencia cardiaca
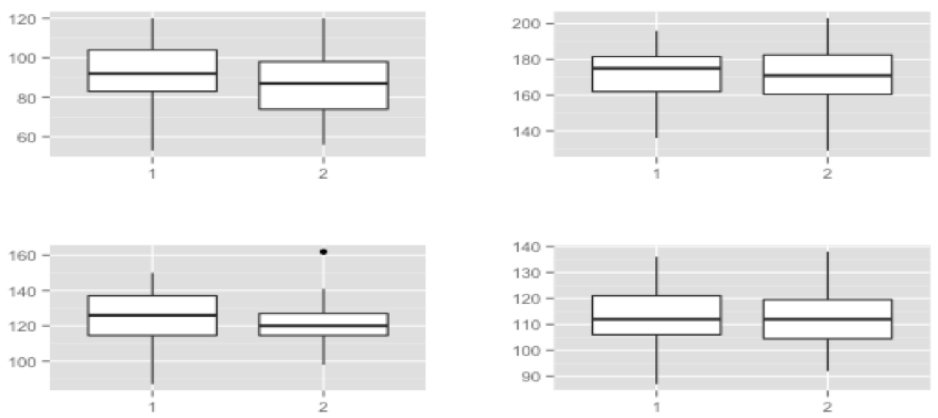

Se describen en el eje Y los valores de frecuencia cardiaca ( $1 / \mathrm{min})$ y en el eje $\mathrm{X}$ el grupo asignado (código 1: sin música y código 2: con música), donde: frecuencia cardiaca antes de la prueba (arriba izquierda), frecuencia cardiaca posterior a la prueba (arriba derecha), frecuencia cardiaca de recuperación a los 3 minutos (abajo izquierda) y frecuencia cardiaca de recuperación a los 6 minutos (abajo derecha) para los dos tratamientos evaluados.

Fuente: elaboración propia. 
Los valores promedios de la presión arterial sistólica $(\mathrm{mmHg})$ antes, durante y después de la prueba de Course-Navette, entre G1 y G2, son presentados en la figura 2. Si bien no se observan diferencias por tratamiento, el grupo G2 (con música) presenta un rango (distancia entre el valor mínimo y máximo) más amplio respecto al grupo G1 (sin música).

Figura 2. Valores de presión arterial sistólica
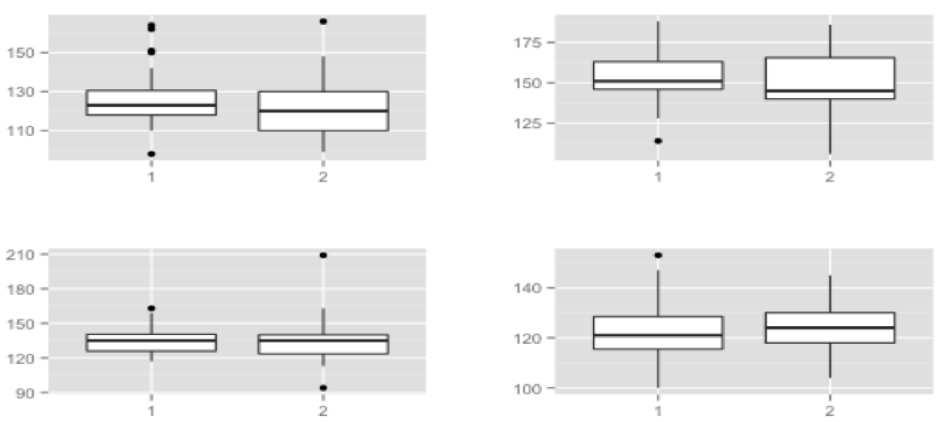

Se describe en el eje Y los valores de presión arterial sistólica ( $\mathrm{mmHg}$ ) y en el eje X el grupo asignado (código 1: sin música y código 2: con música), donde presión arterial sistólica antes de la prueba (arriba izquierda), presión arterial sistólica posterior a la prueba (arriba derecha), presión arterial sistólica a los 3 minutos de recuperación (abajo izquierda) y presión arterial sistólica a los 6 minutos de recuperación (abajo derecha) para los dos tratamientos evaluados.

Fuente: elaboración propia.

Los valores promedios de la presión arterial diastólica $(\mathrm{mmHg})$ antes, durante y después de la prueba de Course-Navette, entre G1 y G2, son presentados en la figura 3. Se destaca que los valores promedios de la variable en G1 y G2 describen una tendencia similar tanto en G1 como en G2. 
Figura 3. Valores presión arterial diastólica
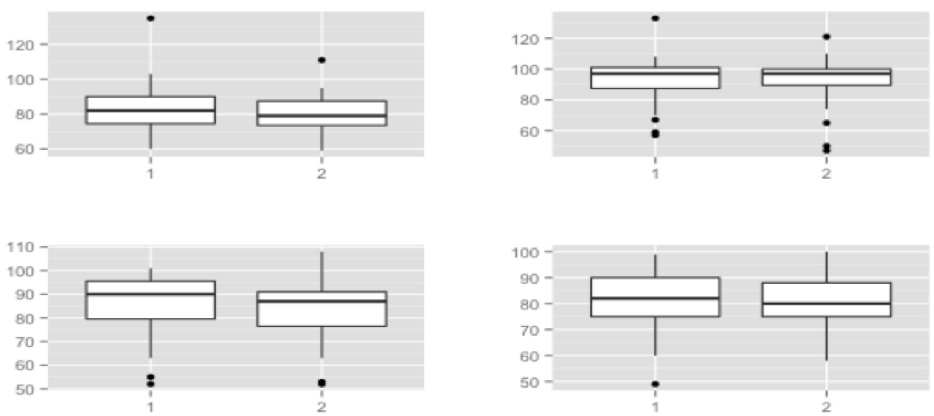

Se describe en el eje Y los valores de presión arterial diastólica $(\mathrm{mmHg})$ y en el eje $\mathrm{X}$ el grupo asignado (código 1: sin música y código 2: con música), donde: presión arterial diastólica antes de la prueba (arriba izquierda), presión arterial diastólica posterior a la prueba (arriba derecha), presión arterial diastólica a los 3 minutos de recuperación (abajo izquierda) y presión arterial diastólica a los 6 minutos de recuperación (abajo derecha) para los dos tratamientos evaluados.

Fuente: elaboración propia.

Se puede consultar los valores de probabilidad p para G1 y G2. Se resalta que solo existen diferencias significativas para la variable "Fase test final" entre los grupos estudiados, es decir, la etapa a la cual llegó cada sujeto fue superior en el grupo G1 respecto a G2 en todas las edades.

Tabla 1. Valores de probabilidad p por variable

\begin{tabular}{|c|c|}
\hline Variable & Valor de significancia $(\mathrm{p})$ \\
\hline FCmáx $(\mathrm{L} / \mathrm{min})$ inicial & $\mathbb{S}$ \\
\hline PAS $(\mathrm{mmHg})$ inicial & $\mathbb{S}$ \\
\hline PAD $(\mathrm{mmHg})$ inicial & $\mathbb{S}$ \\
\hline FCmáx $(1 / \mathrm{min})$ final & $\mathbb{S}$ \\
\hline PAS $(\mathrm{mmHg})$ final & $\mathbb{S}$ \\
\hline PAD $(\mathrm{mmHg})$ final & $* *$ \\
\hline Etapa alcanzada en Course-Navette & $\mathbb{S}$ \\
\hline FCmáx $(1 / \mathrm{min}) 3$ minutos de recuperación & $\mathbb{S}$ \\
\hline PAS $(\mathrm{mmHg}) 3$ minutos de recuperación & $\mathbb{S}$ \\
\hline PAD $(\mathrm{mmHg}) 3$ minutos de recuperación &
\end{tabular}




\begin{tabular}{|c|c|}
\hline Variable & Valor de significancia $(\mathrm{p})$ \\
\hline FCmáx $(1 / \mathrm{min}) 6$ minutos de recuperación & $\mathbb{S}$ \\
\hline PAS $(\mathrm{mmHg}) 6$ minutos de recuperación & $\mathbb{S}$ \\
\hline PAD $(\mathrm{mmHg}) 6$ minutos de recuperación & $\mathbb{S}$ \\
\hline
\end{tabular}

Valores de probabilidad p entre las variables fisiológicas y el grupo asignado (código 1: sin música y código 2: con música). Valores expresados como no significativo (\$); muy significativo $(\mathrm{p}<0.001[* *])$.

\section{Fuente: elaboración propia.}

Al realizar el análisis de la frecuencia cardiaca $(1 / \mathrm{min})$ incluyendo las cuatro fases evaluadas (inicial, final y recuperación a los 3 y 6 minutos), se evidencian diferencias significativas en las fases $(\mathrm{p}<0.05-0.01)$, pero no se observan diferencias significativas entre los dos tratamientos evaluados $(\mathrm{p}=0.191)$ (ver figura 4).

Figura 4. Frecuencia cardiaca por etapas
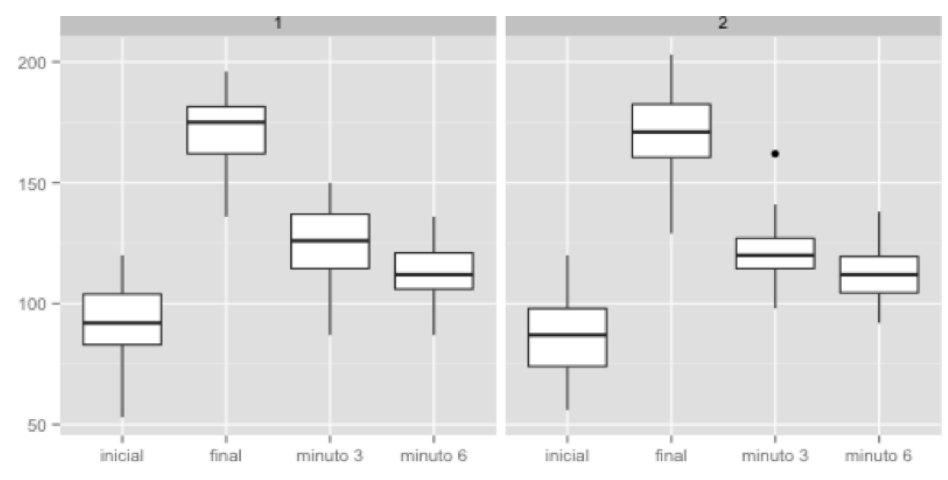

Se describe en el eje Y los valores de la frecuencia cardiaca ( $1 / \mathrm{min})$ y en el eje X la etapa de la prueba por grupo (inicial, final, a los 3 minutos de recuperación, a los 6 minutos de recuperación. Código 1: sin música y código 2: con música). Valores expresados como media \pm desviación estándar.

Fuente: elaboración propia.

Se observa un comportamiento semejante para la PAS ( $\mathrm{mmHg}$ ) incluyendo las cuatro fases evaluadas (inicial, final y recuperación a los 3 y 6 minutos), donde se describen diferencias significativas entre las fases 
$(\mathrm{p}<0.05-0.01)$, pero no se observan diferencias significativas entre los dos tratamientos evaluados $(\mathrm{p}=0.191)$.

Figura 5. Presión arterial sistólica por etapas

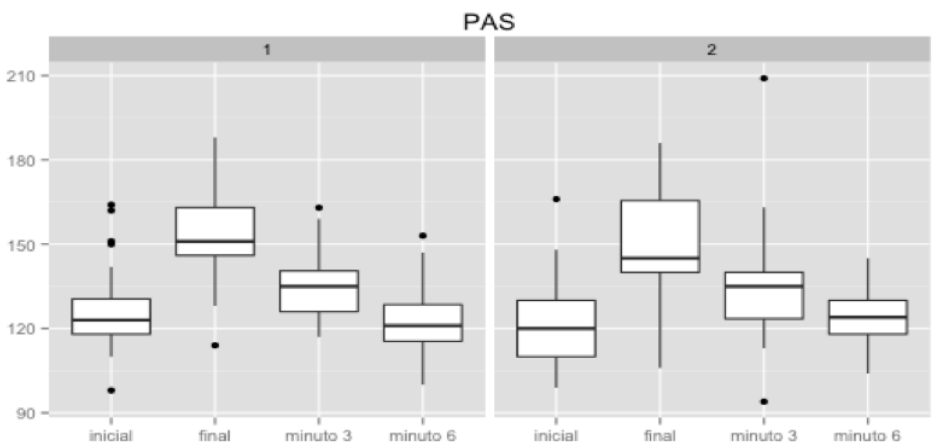

Se describe en el eje Y los valores de la presión arterial sistólica $(\mathrm{mmHg})$ y en el eje $\mathrm{X}$ la etapa de la prueba por grupo (inicial, final, a los 3 minutos de recuperación, a los 6 minutos de recuperación; código 1: sin música y código 2: con música). Valores expresados como media \pm desviación estándar.

Fuente: elaboración propia.

\section{Discusión}

En el presente estudio se evaluó la relación entre música y respuesta cardiovascular anticipatoria, durante el ejercicio y posterior a él. Así mismo, se valoró el rendimiento en pruebas de esfuerzo submáximo con la intervención de la música antes y después del ejercicio.

En estudios previos realizados en condiciones controladas mediante pruebas directas en laboratorios y análisis de los efectos de la música en variables fisiológicas mediante actuaciones anaeróbicas máximas, como los estudios realizados por Jarraya et al. (2012) y Chtourou et al. (2012), se evaluó la producción de potencia en el test de Wingate en jóvenes universitarios. En contraste, el presente estudio tuvo en cuenta el análisis de la música en la respuesta anticipatoria cardiovascular, durante y después del ejercicio. Además, se aplicó en sujetos escolares entre 9 y 17 años una prueba de campo para analizar el componente aeróbico mediante el test de 
Course-Navette. De igual forma, se confirman resultados anteriores sobre aplicabilidad del test de Course-Navette de 20 metros en el ámbito escolar (García y Secchi, 2014), aunque en el presente estudio se aportan evidencias sobre el uso de la música en pruebas aeróbicas.

Los resultados no confirman estadísticamente los efectos de la música en las variables fisiológicas, ya que no hubo diferencias significativas $(\mathrm{p}<0,05)$. Por el contrario, un metaanálisis realizado por Loomba et al., (2012) sobre los efectos de la música en la presión arterial y la frecuencia cardiaca muestra diferencias significativas, una reducción significativamente mayor de la PAS antes y después del ejercicio, así como una reducción significativamente mayor en la PAD. Hay que tener en cuenta que los cambios que conlleva la pubertad pueden influir en los resultados paradójicos respecto a estudios con población adulta, según los cuales la respuesta de la frecuencia cardiaca en niños para una intensidad de ejercicio determinada es mayor que en los adultos (Costill, Wilmore y Kenney, 2012).

Respecto a la frecuencia cardiaca, Watanabe et al. (2015) encontraron que una combinación tempo-ritmo más rápido podría aumentar la FC media. En el presente estudio la FC del grupo G2 (con música) no mostró una reducción significativa en comparación con el grupo G1 (sin música). Sin embargo, las medidas de tendencia central del G2 en las fases 1, 2 y 3 de la prueba evidenciaron una leve reducción en la media respecto al G1, lo cual se puede interpretar como un efecto relajante de la música en la FC del promedio de los sujetos.

Los valores de la FC en las cuatro fases de la prueba muestran una tendencia normal de la actividad nerviosa simpática (ANS) y parasimpática (ANP), es decir, la ANS predomina en el ejercicio, y la ANP predomina en la recuperación. Se encontraron diferencias significativas en las fases de cada grupo G1 y G2, pero no entre los ellos. Sin embargo, la media de G2 fue menor que G1. En este caso se puede decir que la música estimula el sistema nervioso autónomo por igual. En contraste, Watanabe et al. (2015) encontraron que el incremento de la FC media se debe al aumento de la actividad simpática estimulada por el ritmo de la música.

De igual forma, los valores de PAS y PAD del G2 no se vieron afectados significativamente, a diferencia del grupo G1, que no recibió el estímulo musical. Al igual que en la FC, en las medidas de tendencia central la presión arterial (PA) en el ciclo 1 y 2 en PAS y ciclos 1,3 y 6 en PAD, la media 
se ubica un poco más abajo en el G2 y más arriba en G2, lo cual indica una leve reducción de dichos valores en G2. Como ya se dijo, la presión arterial (PA) es un factor que varía en proporción al gasto cardiaco, por tanto, si los valores de FC y volumen sistólico cambian en la adolescencia, de igual forma lo hacen los de la PA.

Por otro lado, se corrobora el efecto ergogénico de la música en el rendimiento físico, discutido en estudios previos como la revisión hecha por Bigliassi, Estanislau, Carneiro, Kanthack y Altimari (2013) sobre música y ayuda ergogénica psicológica durante el ejercicio, en la cual se encontró que el uso de la música como una acción ergogénica ha tenido éxito durante los ejercicios de intensidad submáxima principalmente ( $\leq 70 \%$ VO2 máx). Los resultados hallados en el presente estudio mostraron una diferencia muy significativa en el grupo G2 ( $\mathrm{p}<0.001)$, dado que los sujetos obtuvieron mejores resultados y consiguieron llegar más lejos en la prueba de esfuerzo aeróbico. Lo anterior evidencia que el estímulo musical previo mejora el rendimiento y retarda la fatiga causada por el esfuerzo. Esto sugiere que la música puede ser utilizada como herramienta en las pruebas y competencias de esfuerzos submáximo como el test de Course-Navette en escolares.

Para futuras investigaciones sobre el tema se sugiere hacer estudios longitudinales que analicen el efecto de la música en respuestas fisiológicas en niños y adolescentes y hacer experimentos en los que se implemente la música en el contexto de la formación deportiva infantil.

A modo de conclusión, los resultados del presente estudio son un aporte significativo frente a las evidencias acerca del uso de la música en el contexto de la actividad física y podría ser una herramienta de trabajo muy útil con niños y jóvenes, ya sea en el campo del entrenamiento deportivo o la educación física, como programador de la intensidad del ejercicio y factor ergogénico en el calentamiento y la recuperación del esfuerzo.

\section{Referencias}

Alvero-Cruz, J. R., Gómez, L. C., Ronconi, M., Vázquez, R. F. y Manzañido, J. P. (2011). La bioimpedancia eléctrica como método de estimación de la composición corporal: normas prácticas de utilización. Revista Andaluza de Medicina del Deporte, 4(4), 167-174. 
Arazi, H., Asadi, A. y Purabed, M. (2015). Physiological and Psychophysical Responses to Listening to Music during Warm-Up and Circuit-Type Resistance Exercise in Strength Trained Men. Journal of sports medicine, 1-6. http:// dx.doi.org/10.1155/2015/389831

Bigliassi, M., Estanislau, C., Carneiro, J. G., Kanthack, T. F. D. y Altimari, L. R. (2013). Música: un recurso psicofisiológico para el ejercicio físico y deporte. Archivos de medicina del deporte: revista de la Federación Española de Medicina del Deporte y de la Confederación Iberoamericana de Medicina del Deporte, (157), 311-320.

Bood, R. J., Nijssen, M., van der Kamp, J. y Roerdink, M. (2013). The power of auditory-motor synchronization in sports: enhancing running performance by coupling cadence with the right beats. PloS one, 8(8), e70758.

Caballero Meneses, J. A. y Ménez Díaz, M. (2010). Influencia del tempo de la música en las emociones/influence of music tempo on emotion. Revista Colombiana de Psicología, 19(1), 37-44.

Chtourou, H., Chaouachi, A., Hammouda, O., Chamari, K. y Souissi, N. (2012). Listening to music affects diurnal variation in muscle power output. International Journal of Sports Medicine, 33(1), 43-7.

Costill, D. L., Wilmore, J. H. y Kenney, W. L. (2012). Physiology of sport and exercise. Physiology Of Sport And Exercise-9780736094092-66, 78.

García, G. C. y Secchi, J. D. (2014). Test course navette de 20 metros con etapas de un minuto. Una idea original que perdura hace 30 años. Apunts. Medicina de l’Esport, 49(183), 93-103.

González, J. V., Arenas, O. A. V. y González, V. V. (2012). Semiología de los signos vitales: Una mirada novedosa a un problema vigente:/Vitals sign semiology: the new look to an actual problem. Archivos de Medicina (Manizales), 12(2), 221-240.

Hernández, R., Fernández, C. y Baptista, P. (2006). Metodología de la Investigación. México: McGraw-Hill Interamericana.

Jarraya, M., Chtourou, H., Aloui, A., Hammouda, O., Chamari, K., Chaouachi, A. y Souissi, N. (2012). The effects of music on high-intensity short-term exercise in well trained athletes. Asian journal of sports medicine, 3(4), 233.

Karageorghis, C. I., Jones, L., Priest, D. L., Akers, R. I., Clarke, A., Perry, J. M., Lim, H. B. (2011). Revisiting the relationship between exercise heart rate and music tempo preference. Research quarterly for exercise and sport, 82(2), 274-284. 
Lane, A. M. Davis, P. A., y Devonport, T. J. (2011). Effects of music interventions on emotional states and running performance. Journal of sports science \& medicine, 10(2), 400.

Núñez Fernández, J. (2009). Generador de melodias basado en reglas (tesis de pregrado). Universidad Carlos III de Madrid, España.

Sillero, M. (2005). Medidas Antropométricas. Facultad deficiencias de la actividad física y del deporte (INEF)-Tema, 2, 38-39.

Soria-Urios, G., Duque, P. y García-Moreno, J. M. (2011). Música y cerebro: fundamentos neurocientíficos y trastornos musicales. Revista de Neurología, 52(1), $45-55$.

Watanabe, K., Ooishi, Y. y Kashino, M. (2015). Sympathetic Tone Induced by High Acoustic Tempo Requires Fast Respiration. PloS one, 10(8), e0135589.

Villegas González, J., Villegas Arenas, O. A. y Villegas González, V. (2012). Semiología de los signos vitales: Una mirada novedosa a un problema vigente. Archivos de Medicina, 12(2), 221-240.

Zamorano, G., Peinado Lozano, A. B., Benito Peinado, P. J. y Calderón Montero, F. J. (2013). Respuesta de la frecuencia cardiaca de anticipación y recuperación en función del nivel de entrenamiento aeróbico. Archivos de Medicina del Deporte, 30(4), 202-207. 